\title{
Commentary: New Complications Make Treatment of "Opioid" Overdose Challenging
}

\author{
John F. Peppin 1,2, Joseph V. Pergolizzi Jr.3,4,5, Albert Dahan', Robert B. Raffa ${ }^{3,4,7,8}$ \\ ${ }^{1}$ Marian University College of Osteopathic Medicine (Clinical Adjunct Professor), Indianapolis, IN, USA \\ ${ }^{2}$ Pikeville University College of Osteopathic Medicine (Clinical Professor), Pikeville, KY, USA \\ ${ }^{3}$ Enalare Therapeutics Inc., Princeton, NJ, USA \\ ${ }^{4}$ Neumentum Inc., Summit, NJ, USA \\ ${ }^{5}$ NEMA Research Inc., Naples, FL, USA \\ ${ }^{6}$ Leiden University Medical Center, Leiden, The Netherlands \\ ${ }^{7}$ University of Arizona College of Pharmacy (Adjunct Professor), Tucson, AZ, USA \\ ${ }^{8}$ Temple University School of Pharmacy (Professor Emeritus), Philadelphia, PA, USA \\ Email: robert.raffa@gmail.com
}

How to cite this paper: Peppin, J.F., Pergolizzi Jr., J.V., Dahan, A. and Raffa, R.B. (2020) Commentary: New Complications Make Treatment of "Opioid" Overdose Challenging. Pharmacology \& Pharmacy, 11, 362372 .

https://doi.org/10.4236/pp.2020.1112029

Received: December 2, 2020

Accepted: December 26, 2020

Published: December 29, 2020

Copyright $\odot 2020$ by author(s) and Scientific Research Publishing Inc. This work is licensed under the Creative Commons Attribution International License (CC BY 4.0).

http://creativecommons.org/licenses/by/4.0/

\begin{abstract}
The "opioid crisis" has had a tremendous impact not only on its victims, but also on the practice of medicine, pain patients, and society in general. Unfortunately, efforts to "stem the tide" have not been successful at reducing overdose deaths. Counterbalancing the many ardent efforts to eliminate overdose deaths (such as the current widespread availability and use of opioid-receptor antagonists such as naloxone) is influx of the illicit fentanoids (i.e., fentanyl and analogs). In addition to their high-potency, the fentanoids differ in surprising ways from more "traditional" opioids such as morphine and heroin. This uniqueness contributes to a reduced effectiveness of opioid receptor antagonists in the treatment of opioid overdose. Further greatly complicating overdose treatment is polysubstance abuse (e.g., an opioid plus a benzodiazepine). The non-opioid in the combination is not responsive to an opioid-receptor antagonist, which imparts additional challenges. Thus, the new reality introduces complications that negatively impact efforts to reverse "opioid" overdose. New approaches to improve outcomes in individuals who experience respiratory depression due to fentanoid-induced or polysubstance-induced overdose are needed. Approaches that harmonize with the new reality, perhaps something like a non-opioid "agnostic" pharmacologic ventilatory stimulant, would provide a welcome addition to the current choices.
\end{abstract}

\section{Keywords}

Opioid Overdose, Fentanoid, Naloxone, Polysubstance Abuse, Respiratory Stimulant 


\section{Introduction}

The opioid crisis has profoundly impacted medical practice, pain patients, drug regulatory agencies, legislatures, insurers, the courts, and everyone else. The media, government, and professional organizations decried an over-prescription of, and near-unlimited access to, prescription opioid medications as the source of the problem [1]. Local, County, State, and Federal agencies developed regulations to "stem the tide" of prescription drug overdose [2]. Professional societies were formed to specifically combat the problem, and multiple guidelines were developed and promulgated [3] [4]. The concern was well-placed, but much recrimination and many good-intentioned efforts over-simplified the problem. While legislation and regulatory action was implemented, legal action and financial penalties enforced, professional societies swung the pendulum and become "anti-opioid", overdose deaths continue to increase at a staggering rate [5] [6]. One of the issues is that the reality is changing. Prescription opioids are now a smaller part of the problem (Figure 1), replaced by illicit (and often adulterated) substances; low-potency opioids have been replaced by new high-potency opioids; and overdose is commonly poly-substance, i.e., involves the combination of a non-opioid (such as a benzodiazepine) with the opioid. This new reality is unfortunately revealing an "Achilles heel" of the once nearly always effective opioid receptor antagonists (ORAs) such as naloxone. This is particularly unfortunate, given the recent trend to allow naloxone to be more accessible to patients, their family and other caregivers, and even school officials [7]. We present a succinct overview of the current problem, and speculate on what alternative mechanistic approach might augment the ORA approach.

\section{The Complication of Fentanoids}

Fentanyl was first synthesized and developed by Janssen Pharmaceutica in 1960 [8]. It was developed in order to overcome some of the disadvantages of morphine use in anesthesia. Fentanyl's high potency, high lipid solubility, cardiostability, lack of propensity to cause histamine-release, and relatively short action, make it ideal for surgical anesthesia [9] [10]. Its physicochemical properties gives it the flexibility to be delivered by IV, SC, transdermal, transbuccal/mucosal, and intranasal routes [11]. Unfortunately, some of these same properties make the

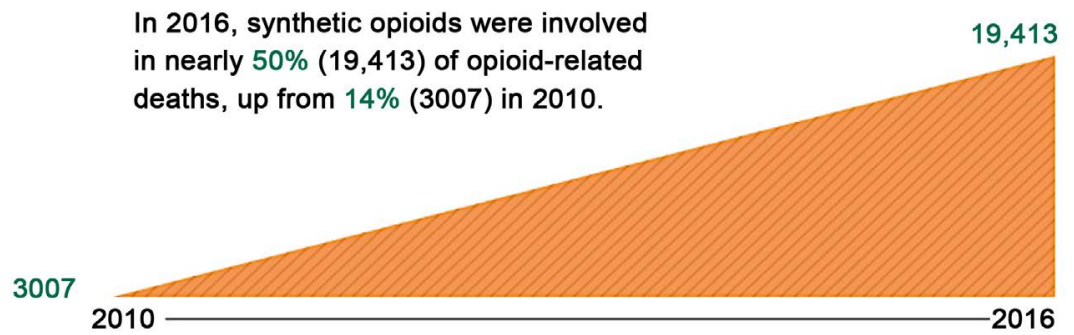

Figure 1. In 2016 synthetic opioids (primarily fentanyl) passed prescription opioids as the most common drugs involved in overdose deaths in the United States. https://www.drugabuse.gov/sites/default/files/infographic-synthetic-opioids.pdf. 
fentanoids (fentanyl and congeners), more "likeable" (Figure 2) [12].

Fentanoids have high binding affinity for the $\mu$ opioid receptor, and lower affinity for the $\delta$ - and $\kappa$-types [13]. They are more potent than morphine, meaning that a lower dose will provide the same level of effect as a higher dose of morphine. Members of the pharmaceutical fentanyl family (e.g., alfentanil, carfentanil, fentanyl, remifentanil, and sufentanil) are used in anesthesia and pain management. Illicitly-manufactured members of the fentanyl family, include illegal congeners ("designer" fentanoids). Multiple illicitly-manufactured fentanoids (IMFs) and "designer" fentanoids are readily available at low cost, often cheaper than alternatives [14].

Because of the physiochemical properties of the fentanoid's, death can occur quickly (due to respiratory depression), especially in users who have not developed tolerance to opioids (actually, for reasons not understood there is very incomplete cross-tolerance between the fentanoids and other opioids) [15]. Whereas a heroin overdose causes death in about 30 minutes, a fentanoid overdose can cause death within only a very few minutes. The former timing provides a window of opportunity for intervention (administration of an ORA, for example), whereas the latter timing results in death before remedial action can be taken. Carfentanil is a fentanoid that is relatively easy and inexpensive to make and is much more potent than fentanyl [16]. It is both legally manufactured as an approved drug, and illegally made for abuse. It is only legally approved in the

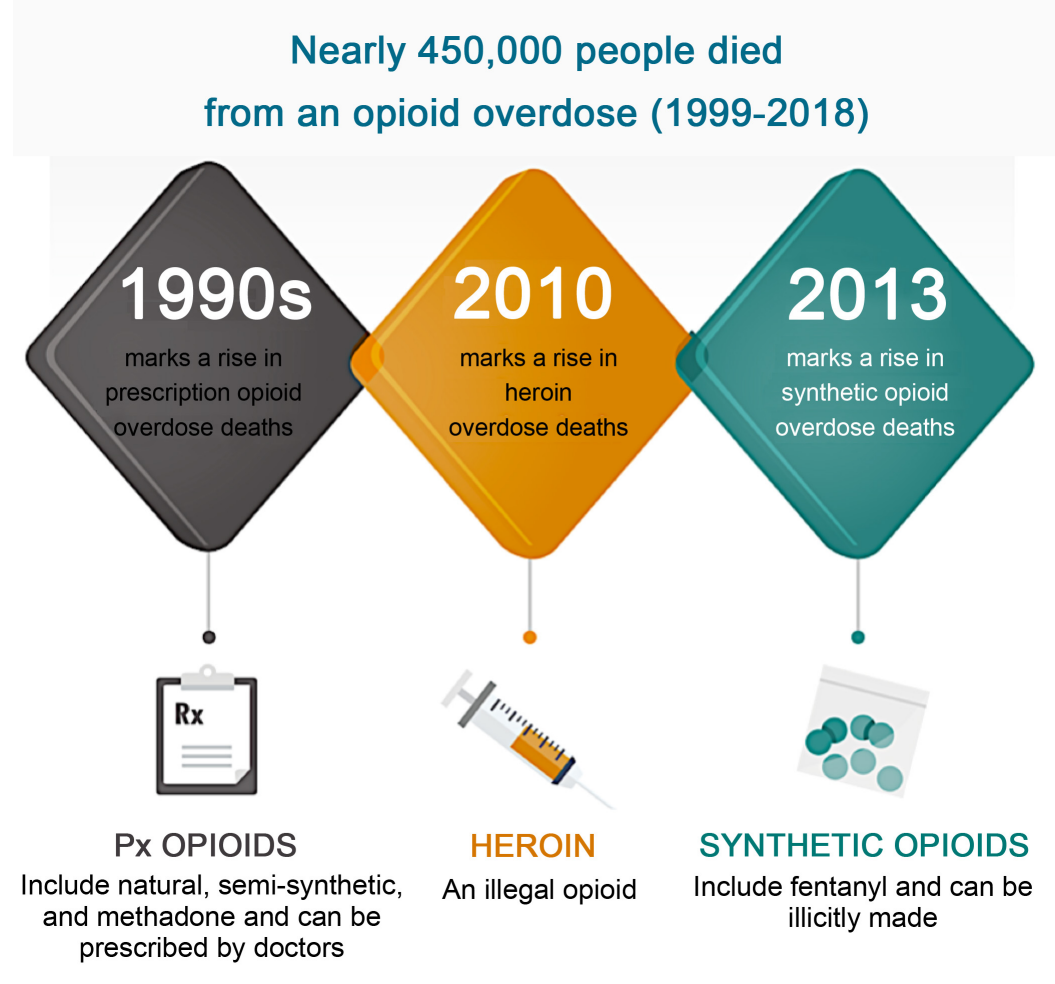

Figure 2. In 2016 synthetic opioids (primarily fentanyl) passed prescription opioids as the most common drugs involved in overdose deaths in the United States. https://www.drugabuse.gov/sites/default/files/infographic-synthetic-opioids.pdf. 
US for veterinary practice. It has a very high binding affinity for the $\mu$-opioid receptor, and has a potential for "re-narcotization", which requires repeat dosing of an ORA to reverse. Fentanyl is approximately 50 to 100 -times more potent than morphine; carfentanil is approximately 10,000-times more potent than morphine [14] [16]. It has been suggested that to treat a carfentanil overdose, "the initial naloxone dose administered should be greater than the maximum empiric naloxone dose recommended by the American Hospital Association" [16]. So it can be possible to reverse fentanoid-induced respiratory depression, even at high doses, but higher doses of the ORA are required, and for a longer time period (possibly even by continuous infusion) until the fentanoid is out of the system.

IMFs, produced in uncontrolled clandestine laboratories, is made from inexpensive and readily-available precursors, often using a fairly simple synthetic method [14]. IMFs are attractive because of high profitability. They are easyavailability on the Internet at a low price, and may escape detection in laboratory tests [14]. IMFs have been made into tablets resembling Xanax, Norco, and oxycodone, and added to cocaine and heroin [17].

\section{The Complication of Atypical Effects of Fentanoids}

Large doses of fentanyl can increase glucose and cause changes in both hippocampal and limbic temperature and metabolic activity [18]. These effects are different than those produced by morphine, heroin, or oxycodone. Amnestic syndromes are also seen with fentanyl overdose, but not with other opioids [19]. Many studies as well as clinical experience alert to cardiovascular complications with fentanyl overdose (including myocardial ischemia, QTc-prolongation and bradycardia), that could lead to fatal outcomes [20]. There have even been platelet effects reported for fentanyl [13]. Polysubstance use is a common finding with fentanoids: alcohol, benzodiazepines, and illicit substances [21]. Many individuals who overdose on these combinations often are unaware that a fentanoid was involved [8] [21]. Due to their high lipid solubility, fentanoids have different binding profiles at opioid receptors that modifies the "agonist:antagonist" ratio, impeding equilibrium competition, which may reduce the ability of naloxone to displace them [22].

\section{The Complication of Polysubstance Overdose}

Reports of polysubstance overdose deaths go back decades. A study of heroin overdose deaths in San Francisco in the 1970s found 47\% positive for ethyl alcohol and $28 \%$ positive for "other drugs" [23]. Some estimates are as high as $80 \%$ [24], involving alcohol, sedatives, benzodiazepines, and illicit drugs [25] [26]. For example, ethanol together with oxycodone causes greater ventilatory depression than does either alone [27]. Benzodiazepines are one of the most commonly-prescribed substances [28], and they are often implicated in opioid-related deaths [29]. Other frequent combinations involve cannabis/cannabi- 
noids, cocaine, and amphetamines [30] [31] [32] [33], although the estimates are highly variable [33]. Those who abuse fentanoids tend to display greater polysubstance abuse, although this may be due to secondary factors such as greater availability, lower cost, or being unaware of the composition of what is being taken [8] [13]. There is also lack of consensus concerning aspects of the appropriate forensic and medico-legal determination of fentanoid overdose death. Even the appropriate site of specimen collection is "still under debate" [34].

\section{The Complication of Naloxone vs Fentanyl and Fentanoids}

It has been reported that "respiratory depression by fentanoids is harder to reverse with naloxone ... and may require multiple and/or higher doses of naloxone." [22]. For other opioids, it used to be common practice to keep the naloxone dose low in order to prevent serious problems with acute precipitated withdrawal. However, a requirement for higher doses of naloxone have become the norm in today's IMF environment. The use of higher doses and multiple doses of naloxone depend of a number of criteria, including regional differences, type of fentanoid and other drugs that are on board. Therefore, it is difficult to establish a "standard" naloxone dosage and dosing schedule in a given overdose setting [35]. Fentanyl use, especially intravenously, has a higher incidence of overdose compared to other opioids. The risk is twice that of heroin, and 8-fold that of oxycodone or morphine [14]. This might result from the fact that fentanoids display "atypical micro-pharmacokinetics" that could affect how naloxone acts at the opioid receptor [22], reducing the ability of naloxone to displace a fentanoid from the receptor [22].

Opioid-induced chest wall rigidity, also called "WoodenChest Syndrome (WCS)", has been known for decades, although it is usually described in the anesthesiology literature related to surgical procedures [8] [36]. WCS presents as musclewall rigidity, laryngospasm, seizure-like events, cyanosis, and loss of consciousness [37] [38]. WCS related to overdose has become more common as the use of illicit fentanoids has increased. WCS can be seen with as little as 50 micrograms of intravenous fentanyl [8]. The incidence has been reported to be anywhere from $8 \%$ to $100 \%$, and has also been reported for alfentanil, norfentanyl, and sufentanil [39] [40]. Overdose death from fentanyl can occur so quickly that a metabolite, norfentanyl, is often not found in toxicologic examination [39]. This has been used as support for the argument that WCS is a cause of fentanoid-induced respiratory depression and overdose death. Not only is WCS not reversed by an ORA - an ORA may make it worse [36]. It has been suggested that fentanoid overdose sets up a potential "double-whammy": WCS and respiratory depression [22]. Recent data suggest that perhaps it is a "triple-whammy": WCS, respiratory depression, and laryngeal spasm. There have been suggestions that WCS is either caused by, or is exacerbated by, naloxone [41]. The mechanism and actual incidence are not known, nor has a clear treatment paradigm been developed [42]. But laryngospasm is almost exclusively reported with lipophilic opio- 
ids, such as the fentanoids [36]. Fentanoids increase norepinephrine levels, which can worsen both chest wall rigidity and laryngospasm [38]. Activation of the recurrent laryngeal and external superior laryngeal nerves are stimulated by sympathetic innervation and norepinephrine release [38]. Motor neurons involved in laryngeal function increase their firing rate within 20 secs of an intravenous fentanyl injection [43]. The stimulation of norepinephrine release by fentanyl is intensified by the blockade of locus coeruleus GABAergic inhibitory neurons [44]. Cholinergic stimulation by fentanyl may also cause reduced perfusion on coronary, hepatic and cerebral systems [40]. This might explain the chest pain and bradycardia seen in fentanyl overdose [44].

Unlike morphine or oxycodone, which in low to moderate doses increases brain oxygen levels through neurovascular compensation, fentanyl produces a dramatic reduction in brain oxygen levels [36]. Fentanyl is 10 to 20 -times more potent in this reduction than is morphine [38]. When fentanyl is used with heroin, the combination produces an even greater reduction in brain oxygen levels [38]. And unlike other opioids, fentanyl reduces both tidal volume and respiratory rate [15].

\section{Looking Ahead: Respiratory Stimulants?}

SinceAnaleptics (central nervous system stimulants) have a long history, but recently there has been a renewed interest as an addition to ORA treatment of drug overdose. Although various agents have been used for decades to stimulate respiration, most of them were used in the surgical suite, had significant side effects, and are no longer used. But due to the conundrum prompted by the popularity of illicit fentanoids and polysubstance abuse, there is a need for alternatives and additives to the apparently diminishingly-effective ORAs. As a result, there has been a resumed interest in respiratory stimulation as a strategy. Such an agent would be "agnostic" to the type of drug inducing the respiratory depression. A number of agents have been, or are being, evaluated. These include modulators of some potassium channel subtypes, serotonin receptor agonists, agents that interact with ionotropic AMPA ( $\alpha$-amino-3-hydroxy-5-methyl-4isoxazolepropionic acid) subtype of glutamate receptor (AMPAkines), and phosphodiesterase inhibitors [45].

Almitrine, (2,4-Bis(allylamino)-6-[4-[bis(p-fluorophenyl)metyl]-1-piperazinyl$\mathrm{s}$-triazine), is an older drug that was shown to produce short-term improvements of respiration in patients with acute respiratory failure [46]. Although it was never approved in the US, and it was removed from the European market due to weight loss and peripheral neuropathy with chronic administration, its mechanism of action is informative. It acts as an agonist at peripheral chemoreceptors expressed on carotid bodies. Its site of action was demonstrated when transection of the carotid sinus nerve blocked the ventilatory effects of almitrine [47]. More specifically, almitrine inhibits $\mathrm{Ca}^{2+}$-dependent $\mathrm{K}^{+}$channels without altering voltage-dependent $\mathrm{K}^{+}, \mathrm{Na}^{+}$, or calcium currents [48]. 
Doxapram,1-Ethyl-4-(2-morpholin-4-ylethyl)-3,3-diphenyl-pyrrolidin-2-one, was developed in the 1960s, and is still available for use as an analeptic respiratory stimulant [49]. Precautions are needed for certain patient populations [50]. Doxapram is a potent inhibitor of TWIK(Tandem of P-domains in a Weakly Inward rectifying $\mathrm{K}^{+}$channel)-related acid-sensitive $\mathrm{K}^{+}$-channels (TASK-1 and TASK-3) with an action on the carotid body [49] [51].

ENA-001 (formerly GAL-021), 2-[( $N$-Methyl- $N$-methoxy)amino]-4,6bis(propylamino)-1,3,5-triazine, also acts on select potassium channels in the carotid body, but in a different way, and on a different part of the homeostatis feedback loop than does almitrine and doxapram. It inhibits large-conductance $\mathrm{Ca}^{2+} /$ voltage-activated $\mathrm{K}^{+}$channels, $\mathrm{BK}_{\mathrm{Ca}}$ (also known as Maxi-K, KCNMA1, Slo1), primarily working through the carotid body [52] [53]. In human volunteers under isohypercapnic conditions, a separation between ENA-001 and placebo on minute ventilation was observed at low-dose alfentanil plus high-dose ENA-001 and high-dose-alfentanil plus high-dose ENA-001. Similar observations were made on poikilocapnic ventilation and arterial $\mathrm{pCO}_{2}[54]$.

\section{Conclusions}

The current climate of fentanoid overdose presents significant complications for successful treatment, partly because fentanoids have unique features different from other opioids that are abused. Some known and suspected differences include:

- Higher potency,

- Faster onset,

- Complex competition with naloxone at the opioid receptor,

- Changes in brain glucose levels and temperature,

- Cardiovascular effects,

- Wooden chest syndrome,

- Laryngeal spasm,

- Reduced brain oxygenation.

These all may contribute to fentanoid-induced deaths and the resistance seen to standard receptor-antagonist therapies such as naloxone. These cases are further complicated by the common occurrence of polypharmacy. They can be very complicated and require close monitoring and aggressive treatment.

With other, even more highly potent, fentanoids on the horizon [55], there is a need for additional therapies with novel mechanisms of action that could supplement efforts to reverse acute respiratory depression. Naloxone will not likely be replaced in the near future, but, unfortunately, in spite of its increased use and availability, alone this treatment may not be enough. The use of non-opioid derived medications, such as respiratory stimulants, might be a welcome possible new approach.

\section{Conflicts of Interest}

The authors declare no conflicts of interest regarding the publication of this paper. 


\section{References}

[1] Manchikanti, L., et al. (2012) Opioid Epidemic in the United States. Pain Physician, 15, ES9-ES38.

[2] Jones, M.R., et al. (2018) A Brief History of the Opioid Epidemic and Strategies for Pain Medicine. Pain and Therapy, 7, 13-21.

https://doi.org/10.1007/s40122-018-0097-6

[3] PROP (2020) Physicians for Responsible Opioid Prescribing. http://www.supportprop.org

[4] CDC (2020) CDC Guideline for Prescribing Opioids for Chronic Pain. https://www.cdc.gov/drugoverdose/prescribing/guideline.html

[5] NIDA (2020) Overdose Death Rates. https://www.drugabuse.gov/drug-topics/trends-statistics/overdose-death-rates

[6] NIDA (2020) Opioid Overdose Reversal with Naloxone (Narcan, Evzio). https://www.drugabuse.gov/drug-topics/opioids/opioid-overdose-reversal-naloxone -narcan-evzio

[7] White, N.D. (2019) Increasing Naloxone Access and Use to Prevent Opioid Overdose Death and Disability. American Journal of Lifestyle Medicine, 13, 33-35. https://doi.org/10.1177/1559827618803874

[8] Stanley, T.H. (2014) The Fentanyl Story. The Journal of Pain, 15, 1215-1226. https://doi.org/10.1016/j.jpain.2014.08.010

[9] Suzuki, J. and El-Haddad, S. (2017) A Review: Fentanyl and Non-Pharmaceutical Fentanyls. Drug and Alcohol Dependence, 171, 107-116. https://doi.org/10.1016/j.drugalcdep.2016.11.033

[10] Peng, P.W. and Sandler, A.N. (1999) A Review of the Use of Fentanyl Analgesia in the Management of Acute Pain in Adults. Anesthesiology, 90, 576-599.

https://doi.org/10.1097/00000542-199902000-00034

[11] Darwish, M., Kirby, M., Robertson, P., Tracewell, W. and Jiang, J.G. (2007) Absolute and Relative Bioavailability of Fentanyl Buccal Tablet and Oral Transmucosal Fentanyl Citrate. The Journal of Clinical Pharmacology, 47, 343-350. https://doi.org/10.1177/0091270006297749

[12] Raffa, R.B., et al. (2018) The Fentanyl Family: A Distinguished Medical History Tainted by Abuse. Journal of Clinical Pharmacy and Therapeutics, 43, 154-158. https://doi.org/10.1111/jcpt.12640

[13] Han, Y., et al. (2019) The Rising Crisis of Illicit Fentanyl Use, Overdose, and Potential Therapeutic Strategies. Translational Psychiatry, 9, 282.

https://doi.org/10.1038/s41398-019-0625-0

[14] Karila, L., et al. (2019) New Synthetic Opioids: Part of a New Addiction Landscape. Neuroscience \& Biobehavioral Reviews, 106, 133-140. https://doi.org/10.1016/j.neubiorev.2018.06.010

[15] Hill, R., Santhakumar, R., Dewey, W., Kelly, E. and Henderson, G. (2020) Fentanyl Depression of Respiration: Comparison with Heroin and Morphine. British Journal of Pharmacology, 177, 254-266. https://doi.org/10.1111/bph.14860

[16] Leen, J.L.S. and Juurlink, D.N. (2019) Carfentanil: A Narrative Review of Its Pharmacology and Public Health Concerns. Canadian Journal of Anesthesia, 66, 414-421. https://doi.org/10.1007/s12630-019-01294-y

[17] DEA (2016) Counterfeit Prescription Pills Containing Fentanyls: A Global Threat (DEA-DCT-DIB-021-16). 
https://www.dea.gov/sites/default/files/docs/Counterfeit\%2520Prescription\%2520Pi $\underline{\text { lls.pdf }}$

[18] Kiyatkin, E.A. (2019) Respiratory Depression and Brain Hypoxia Induced by Opioid Drugs: Morphine, Oxycodone, Heroin, and Fentanyl. Neuropharmacology, 151, 219-226. https://doi.org/10.1016/j.neuropharm.2019.02.008

[19] Barash, J.A., et al. (2018) Acute Amnestic Syndrome Associated with Fentanyl Overdose. New England Journal of Medicine, 378, 1157-1158. https://doi.org/10.1056/NEJMc1716355

[20] Locatelli, C., Lonati, D. and Petrolini, V. (2020) New Drugs of Abuse and Cardiovascular Function. In: Brain and Heart Dynamics, Springer, Berlin, 843-868. https://doi.org/10.1007/978-3-030-28008-6 55

[21] Claridge, H., Williams, B. and Copeland, C. (2020) A Deadly Trend in Fentanyl Fatalities (England, 1998-2017). British Journal of Clinical Pharmacology, 86, 437-444. https://doi.org/10.1111/bcp.14135

[22] Gill, H., Kelly, E. and Henderson, G. (2019) How the Complex Pharmacology of the Fentanyls Contributes to Their Lethality. Addiction, 114, 1524-1525. https://doi.org/10.1111/add.14614

[23] Baselt, R.C., Allison, D.J., Wright, J.A., Scannell, J.R. and Stephens, B.G. (1975) Acute Heroin Fatalities in San Francisco. Demographic and Toxicologic Characteristics. Western Journal of Medicine, 122, 455-458.

[24] Taylor, A., Frischer, M. and Goldberg, D. (1996) Non-Fatal Overdosing Is Related to Polydrug Use in Glasgow. BMJ, 313, 1400-1401.

https://doi.org/10.1136/bmj.313.7069.1400b

[25] Gudin, J.A., Mogali, S., Jones, J.D. and Comer, S.D. (2013) Risks, Management, and Monitoring of Combination Opioid, Benzodiazepines, and/or Alcohol Use. Postgraduate Medicine, 125, 115-130. https://doi.org/10.3810/pgm.2013.07.2684

[26] Calcaterra, S., Glanz, J. and Binswanger, I.A. (2013) National Trends in Pharmaceutical Opioid Related Overdose Deaths Compared to Other Substance Related Overdose Deaths: 1999-2009. Drug and Alcohol Dependence, 131, 263-270. https://doi.org/10.1016/j.drugalcdep.2012.11.018

[27] van der Schrier, R., et al. (2017) Influence of Ethanol on Oxycodone-Induced Respiratory Depression: A Dose-Escalating Study in Young and Elderly Individuals. Anesthesiology, 126, 534-542. https://doi.org/10.1097/ALN.0000000000001505

[28] Guina, J., Merrill, B. and LeQuang, J. (2020) Benzodiazepine Therapy: The Good, the Bad, and the Ugly. In: Peppin, J., Pergolizzi, J., Raffa, R. and Wright, S., Eds., The Benzodiazepine Crisis: The Ramifications of an Over-Used Drug Class, Oxford University Press, Oxford, Ch. 3, 17-40.

[29] Peppin, J. and Wright, S. (2020) Benzodiazepines and Pain Management. In: Peppin, J., Pergolizzi, J., Raffa, R. and Wright, S., Eds., The Benzodiazepine Crisis. The Ramifications of an Over-Used Drug Class, Oxford University Press, Oxford, Ch. 9, 149-159.

[30] Connor, J.P., et al. (2013) Polysubstance Use in Cannabis Users Referred for Treatment: Drug Use Profiles, Psychiatric Comorbidity and Cannabis-Related Beliefs. Front Psychiatry, 4, 79. https://doi.org/10.3389/fpsyt.2013.00079

[31] Leri, F., Bruneau, J. and Stewart, J. (2003) Understanding Polydrug Use: Review of Heroin and Cocaine Co-Use. Addiction, 98, 7-22.

https://doi.org/10.1046/j.1360-0443.2003.00236.x

[32] Palamar, J.J., Le, A. and Mateu-Gelabert, P. (2018) Not Just Heroin: Extensive Po- 
lysubstance Use among US High School Seniors Who Currently Use Heroin. Drug and Alcohol Dependence, 188, 377-384.

https://doi.org/10.1016/j.drugalcdep.2018.05.001

[33] Crummy, E., O’Neal, T., Baskin, B. and Ferguson, S. (2020) One Is Not Enough: Understanding and Modeling Polysubstance Use. Frontiers in Neuroscience, 14, 1-27. https://doi.org/10.3389/fnins.2020.00569

[34] Giorgetti, A., Centola, C. and Giorgetti, R. (2017) Fentanyl Novel Derivative-Related Deaths. Human Psychopharmacology, 32, e2605. https://doi.org/10.1002/hup.2605

[35] Peppin, J., Pergolizzi, J.V. and Raffa, R.B. (2020) Are Opioid Receptor Antagonists Adequate for "Opioid" Overdose in a Changing Reality? Journal of Clinical Pharmacy and Therapeutics.

[36] Torralva, R. and Janowsky, A. (2019) Noradrenergic Mechanisms in Fentanyl-Mediated Rapid Death Explain Failure of Naloxone in the Opioid Crisis. Journal of Pharmacology and Experimental Therapeutics, 371, 453-475. https://doi.org/10.1124/jpet.119.258566

[37] Somerville, N.J., et al. (2017) Characteristics of Fentanyl Overdose-Massachusetts, 2014-2016. Morbidity and Mortality Weekly Report, 66, 382-386.

https://doi.org/10.15585/mmwr.mm6614a2

[38] Davis, M.P. and Behm, B. (2020) Reasons to Avoid Fentanyl. Annals of Palliative Medicine, 9, 611-624. https://doi.org/10.21037/apm.2020.01.12

[39] Burns, G., DeRienz, R.T., Baker, D.D., Casavant, M. and Spiller, H.A. (2016) Could Chest Wall Rigidity Be a Factor in Rapid Death from Illicit Fentanyl Abuse? Clinical Toxicology (Philadelphia, Pa.), 54, 420-423.

https://doi.org/10.3109/15563650.2016.1157722

[40] Chou, R., et al. (2017) Management of Suspected Opioid Overdose with Naloxone in Out-of-Hospital Settings: A Systematic Review. Annals of Internal Medicine, 167, 867-875. https://doi.org/10.7326/M17-2224

[41] Levine, R., Veliz, S. and Singer, D. (2020) Wooden Chest Syndrome: Beware of Opioid Antagonists, Not Just Agonists. The American Journal of Emergency Medicine, 38, 411.e415-411.e416. https://doi.org/10.1016/j.ajem.2019.09.009

[42] Trujillo, C., et al. (2020) Objective Characterization of Opiate-Induced Chest Wall Rigidity. Cureus, 12, e8459. https://doi.org/10.7759/cureus.8459

[43] Lalley, P.M. (2003) Mu-Opioid Receptor Agonist Effects on Medullary Respiratory Neurons in the Cat: Evidence for Involvement in Certain Types of Ventilatory Disturbances. American Journal of Physiology-Regulatory, Integrative and Comparative Physiology, 285, R1287-R1304. https://doi.org/10.1152/ajpregu.00199.2003

[44] Breton-Provencher, V. and Sur, M. (2019) Active Control of Arousal by a Locus Coeruleus GABAergic Circuit. Nature Neuroscience, 22, 218-228.

https://doi.org/10.1038/s41593-018-0305-Z

[45] Cotten, J.F. (2014) The Latest Pharmacologic Ventilator. Anesthesiology, 121, 442444. https://doi.org/10.1097/ALN.0000000000000368

[46] Reyes, A., Lopez-Messa, J.B. and Alonso, P. (1987) Almitrine in Acute Respiratory Failure. Effects on Pulmonary Gas Exchange and Circulation. Chest, 91, 388-393. https://doi.org/10.1378/chest.91.3.388

[47] de Backer, W., Bogaert, E., Van Maele, R. and Vermeire, P. (1983) Effect of Almitrine Bismesylate on Arterial Blood Gases and Ventilatory Drive in Patients with Severe Chronic Airflow Obstruction and Bilateral Carotid Body Resection. European Journal of Respiratory Diseases. Supplement, 126, 239-242. 
[48] Lopez-Lopez, J.R., Perez-Garcia, M.T., Canet, E. and Gonzalez, C. (1998) Effects of Almitrine Bismesylate on the Ionic Currents of Chemoreceptor Cells from the Carotid Body. Molecular Pharmacology, 53, 330-339.

https://doi.org/10.1124/mol.53.2.330

[49] Cunningham, K.P., MacIntyre, D.E., Mathie, A. and Veale, E.L. (2020) Effects of the Ventilatory Stimulant, Doxapram on Human TASK-3 (KCNK9, K2P9.1) Channels and TASK-1 (KCNK3, K2P3.1) Channels. Acta Physiologica, 228, e13361.

https://doi.org/10.1111/apha.13361

[50] FDA (2020) Dopram Injection. https://www.accessdata.fda.gov/drugsatfda docs/label/2005/14879s044lbl.pdf

[51] Mitchell, R.A. and Herbert, D.A. (1975) Potencies of Doxapram and Hypoxia in Stimulating Carotid-Body Chemoreceptors and Ventilation in Anesthetized Cats. Anesthesiology, 42, 559-566. https://doi.org/10.1097/00000542-197505000-00009

[52] McLeod, J.F., et al. (2014) GAL-021, a New Intravenous BKCa-Channel Blocker, Is Well Tolerated and Stimulates Ventilation in Healthy Volunteers. British Journal of Anaesthesia, 113, 875-883. https://doi.org/10.1093/bja/aeu182

[53] Roozekrans, M., et al. (2015) Reversal of Opioid-Induced Respiratory Depression by BK-Channel Blocker GAL021: A Pharmacokinetic-Pharmacodynamic Modeling Study in Healthy Volunteers. Clinical Pharmacology \& Therapeutics, 97, 641-649. https://doi.org/10.1002/cpt.99

[54] Roozekrans, M., et al. (2014) Two Studies on Reversal of Opioid-Induced Respiratory Depression by BK-Channel Blocker GAL021 in Human Volunteers. Anesthesiology, 121, 459-468. https://doi.org/10.1097/ALN.0000000000000367

[55] CDC (2020) Synthetic Opioid Overdose Data. https://www.cdc.gov/drugoverdose/data/fentanyl.html 